\title{
Extração de Parâmetros para Simulação de Redes em Aplicativos Móveis de Grande Utilização
}

\author{
Renê Eduardo Pereira Cardozo, Daniel Macêdo Batista \\ ${ }^{1}$ Departamento de Ciência da Computação - Universidade de São Paulo (USP) \\ São Paulo - SP - Brasil \\ rene.cardozodusp.br, batista@ime.usp.br
}

\begin{abstract}
This paper presents an investigation into the adherence of the new transport layer protocol, QUIC, in high used mobile applications and its main network traffic characteristics. For that purpose, 9 applications - each with over 1 billion downloads at Play Store - were chosen. Their network traffic was captured during 5 minutes of usage through the software Wireshark. Based on the traffic characteristics obtained (delay, transferred bytes, number of transferred packets, transfer rate, packet size and IP addresses involved in the communication), parameters were extracted, which can be useful to simulate these aplications in network simulators, such as the $n s-3$.
\end{abstract}

Resumo. Este artigo apresenta uma investigação a respeito da aderência do novo protocolo da camada de transporte, QUIC, por aplicações de grande utilização em dispositivos móveis e suas principais características de tráfego de rede. Para isso, foram escolhidos nove aplicativos com mais de 1 bilhão de downloads cada na Play Store, e eles tiveram seu tráfego de rede capturado durante cinco minutos de utilização por meio do software Wireshark. Baseado nas características encontradas (latência, bytes transferidos, quantidade de pacotes transferidos, taxa de transferência, tamanho dos pacotes e endereços IP envolvidos na comunicação), foram extraídos parâmetros que podem ajudar na simulação destas aplicações em simuladores de rede como o $\mathrm{ns}$-3.

\section{Introdução}

O novo protocolo da camada de transporte QUIC, padronizado pela RFC9000 [Iyengar and Thomson 2021] em maio de 2021 e em fases de testes desde 2012 [Chromium 2013], busca superar o desempenho do TCP e prover adaptabilidade da camada de transporte para cenários contemporâneos e futuros. Esta flexibilidade para diferentes situações é alcançada utilizando o protocolo UDP como base, deferindo o projeto da sua funcionalidade a uma camada acima do sistema operacional.

O QUIC foi proposto considerando o domínio dos dispositivos móveis, Internet das Coisas e redes sem fio, características essas que entram em conflito com a arquitetura do TCP, a qual possui quase 40 anos desde sua padronização [Postel 1981]. Oscilações de conexões em redes sem fio, interferência eletromagnética e obstáculos físicos, causados pela mobilidade do usuário e pela reutilização de canais dentro da área de cobertura de um ponto de acesso, constantemente causam perdas de conexão [Daigavhane and Chawhan 2018] por serem mal interpretadas pelos algoritmos de 
controle de congestionamento do TCP. Além disso, um fator crucial para tecnologias móveis é a eficiência energética, que não provocou preocupação no projeto inicial do TCP.

Tendo como foco a utilização de dispositivos móveis e frente à recente padronização do QUIC, este artigo investiga a sua presença em aplicações de grande utilização em smartphones com sistema operacional Android. Busca também reconhecer as características dos serviços que escolheram utilizá-lo como substituto para o TCP e o perfil do tráfego de pacotes, visando incorporar as aplicações em simulações que comparem os dois protocolos. Com a obtenção das características do tráfego, espera-se aumentar o realismo de simulações, principalmente com o protocolo QUIC.

É importante destacar que o uso de recursos de simulação para redes de computadores permite o estudo de cenários de maneira flexível e com baixo custo. Parâmetros utilizados para a simulação de outros protocolos de transporte, como TCP e UDP, não são totalmente adequados para ambientes que utilizam o QUIC, uma vez que este último pode possuir uma grande variedade de implementações. Além disso, obter parâmetros de base para simulações garante consistência e adequação a cenários reais. Logo, a avaliação do comportamento de aplicações reais se faz necessária. Todos esses aspectos servem de motivação para a pesquisa apresentada neste artigo. Em particular, o simulador ns-3 utiliza os parâmetros de latência e taxa de transferência para definir os canais de comunicação entre dispositivos. Os módulos de aplicações e rede, permitem o envio de pacotes de maneira controlada, baseando-se em seu tamanho e taxa de envio. Estes quatro parâmetros citados, em conjunto com o tamanho total da transmissão e com os algoritmos de perda, erro e mobilidade do próprio simulador, podem ser utilizados para definir cenários de base para a simulação no contexto de dispositivos móveis.

Com relação ao estado da arte, este artigo avança os trabalhos sobre o QUIC relacionados com simulação [Couto et al. 2018] e emulação [Bulgarella et al. 2019] pelo fato de considerar versões atuais do protocolo, algo importante, dado que diversas mudanças foram realizadas no protocolo nos últimos anos. O artigo também avança os trabalhos sobre o QUIC relacionados com medição [Rüth et al. 2018] pelo fato de focar em comunicações de dispositivos móveis e por considerar os aplicativos mais utilizados, escolhas importantes por conta da grande quantidade de tráfego gerado na Internet por esses dispositivos e aplicativos.

O restante deste artigo está organizado da seguinte forma: A Seção 2 explica como foram realizadas as capturas de tráfego e apresenta algumas características preliminares relativas à aderência ao QUIC pelas aplicações. A Seção 3 avança na análise descrevendo diversas características do tráfego capturado. A Seção 4 conclui o artigo e apresenta sugestões de trabalhos futuros.

\section{Capturas e Perfil do Tráfego de Rede}

Foram realizadas capturas de cinco minutos do tráfego de nove aplicações, sendo elas: Youtube, Facebook, Google Maps, Instagram, Youtube Music, LinkedIn, Netflix, Spotify e Whatsapp Messenger. Todos os aplicativos foram baixados da loja de aplicativos oficial do sistema operacional Android. Realizou-se uma navegação casual em todos os aplicativos, utilizando seus recursos mais comuns durante toda a captura. A Tabela 1 lista as versões de cada um dos aplicativos executados.

Os aplicativos foram executados no smartphone Xiaomi Mi Note 10, com sistema 


\begin{tabular}{|c|c|}
\hline Aplicativo & Versão \\
\hline Youtube & 16.14 .35 \\
\hline Facebook & 315.0 .0 .47 .113 \\
\hline Google Maps & 10.65 .3 \\
\hline Instagram & 184.0 .0 .30 .117 \\
\hline Youtube Music & 4.24 .51 \\
\hline LinkedIn & 4.1 .531 \\
\hline Netflix & 7.100 .0 \\
\hline Spotify & 8.6 .18 .720 \\
\hline WhatsApp Messenger & 2.21 .9 .10 \\
\hline
\end{tabular}

Tabela 1. Versão de cada aplicativo

operacional Android na versão 10 QKQ1.190825.002, conectado pelo recurso de Wi-Fi Hotspot a um computador que estava compartilhando a conexão à Internet por uma placa de rede Intel AC 9560 em configuração de ponte. O computador, por sua vez, estava conectado à Internet por meio de uma rede cabeada com capacidade de $240 \mathrm{Mbps}$ de download e 100 Mbps de upload. A máquina possuía um processador Intel Core i79750H, 64GB de memória RAM e sistema operacional Pop! OS 20.04.

Como é possível observar pela Figura 1, que exibe a distribuição dos bytes transferidos por cada protocolo da camada de transporte, serviços de consumo de vídeos e imagens são os mais comuns novos adeptos do QUIC. O aplicativo Netflix, maior representante de streaming de mídia, é uma exceção importante a esta tendência. Segundo pesquisas anteriores, seu protocolo de streaming, o DASH, tem seu desempenho degradado pelo uso do QUIC quando comparado ao TCP [Bhat et al. 2017]. Esse comportamento é repetido com o serviço de streaming de músicas, Spotify, no qual há somente a utilização do TCP. Contudo, outros experimentos e análises da utilização do QUIC para a criação de protocolos de streaming sugerem uma diminuição no tempo de transmissão utilizando o protocolo [Szabó et al. 2016, Choi et al. 2017, Palmer et al. 2018], o que pode indicar uma tendência futura para a sua maior utilização neste cenário.

Pela Figura 2, que exibe os tamanhos máximos dos pacotes observados em intervalos de 1 segundo, temos que o tamanho máximo dos pacotes para todos os serviços é de 1589 bytes, sendo este o padrão obtido para o TCP. Contudo, observando apenas os pacotes utilizando o QUIC, foi constatado que, para os serviços Facebook e Instagram, há um máximo de 1274 bytes por pacote, enquanto serviços como Youtube, Youtube Music e Google Maps possuem um máximo de 1392 bytes. Esta diferença bastante significativa entre os tamanhos máximos dos pacotes evidencia a flexibilidade do protocolo. Diferentes empresas podem possuir implementações distintas do protocolo e, assim, obterem uma alta capacidade de negociação de parâmetros que mais se adequem às características de seu conteúdo e rede interna.

Também é possível identificar, pela Figura 2, a grande diferença de comportamento do aplicativo Netflix, o qual, durante grande parte do intervalo, atinge o tamanho máximo permitido pelo TCP. Ainda, o serviço de streaming possui uma média cerca de duas vezes maior do que a média das outras aplicações. Por conta da discrepância da aplicação em relação às outras, e buscando comparações mais justas, decidiu-se por restringir 


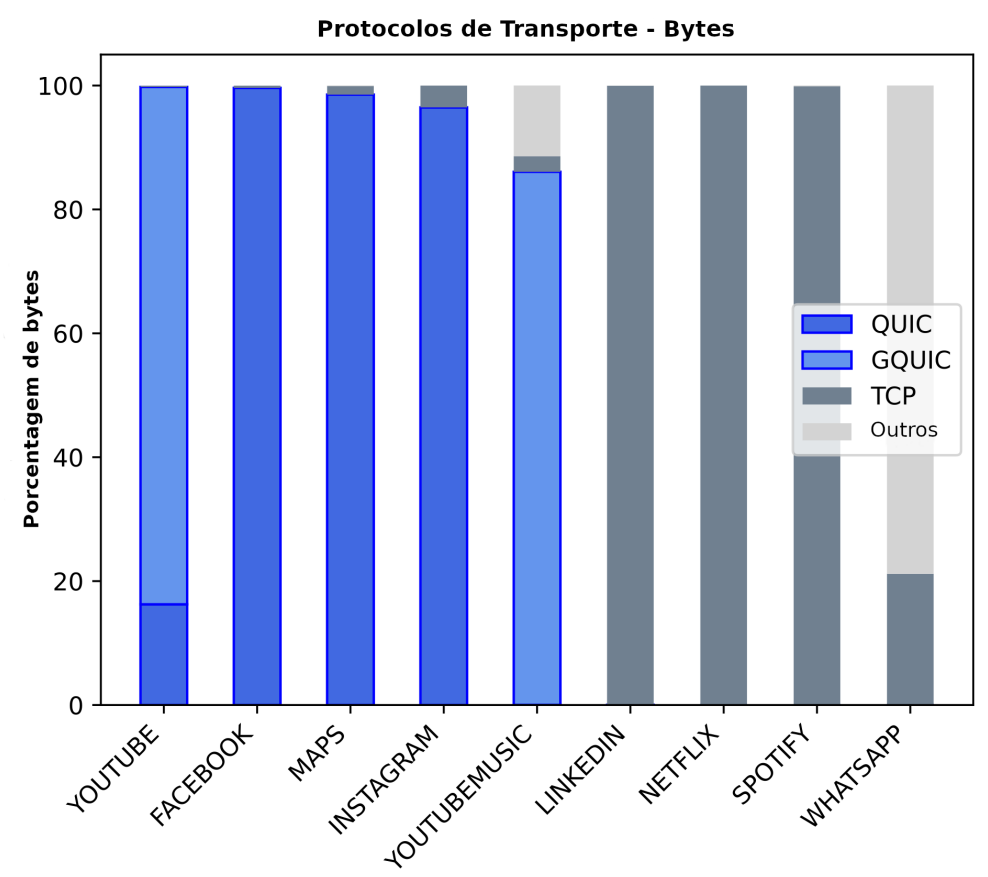

Figura 1. Porcentagem de bytes transmitidos e recebidos por cada protocolo da camada de transporte (gQUIC representa a variação do protocolo QUIC implementada pela Google em alguns serviços).

o escopo da análise aos outros oito aplicativos no restante deste artigo.

Tendo como foco os outros serviços, pode-se perceber uma característica de rajadas em suas transmissões, com picos de transmissão separados por intervalos de pouca ou nenhuma utilização da rede. Este perfil de tráfego é útil para economia de energia na utilização dos recursos de rede, os quais podem consumir cerca de $46 \%$ a mais de energia se optarem por uma taxa de transmissão contínua [Qian et al. 2011].

A Figura 3 mostra o número de pacotes transmitidos pelo aplicativo Youtube a cada $500 \mathrm{~ms}$, onde fica bastante evidente a característica de transmissão em rajadas. Há picos periódicos de transmissão de aproximadamente dois segundos, separados por intervalos periódicos de cerca de cinco segundos. Este padrão se repete em todas as outras sete aplicações, que possuem intervalos de transmissão similares.

\section{Análise do Tráfego}

A partir das capturas, foi possível obter valores para: número de pacotes trocados; número de bytes trocados; taxa de transferência; média do tamanho dos pacotes; média da taxa de pacotes por segundo; e número de endereços IP distintos, que estão resumidos na Figura 4.

É possível observar que, exceto para o Google Maps, as aplicações que utilizam o protocolo QUIC possuem um fluxo de rede maior do que as aplicações que continuam a utilizar o TCP, tendo uma maior taxa de transferência de pacotes por segundo e um maior número de bytes transmitidos.

O comando ping, executado no smartphone utilizando o aplicativo emulador de terminal Termux na versão 0.101 , foi usado para avaliar a latência para o endereço IP 
Tamanho máximo dos pacotes por intervalo de $1 \mathrm{~s}$

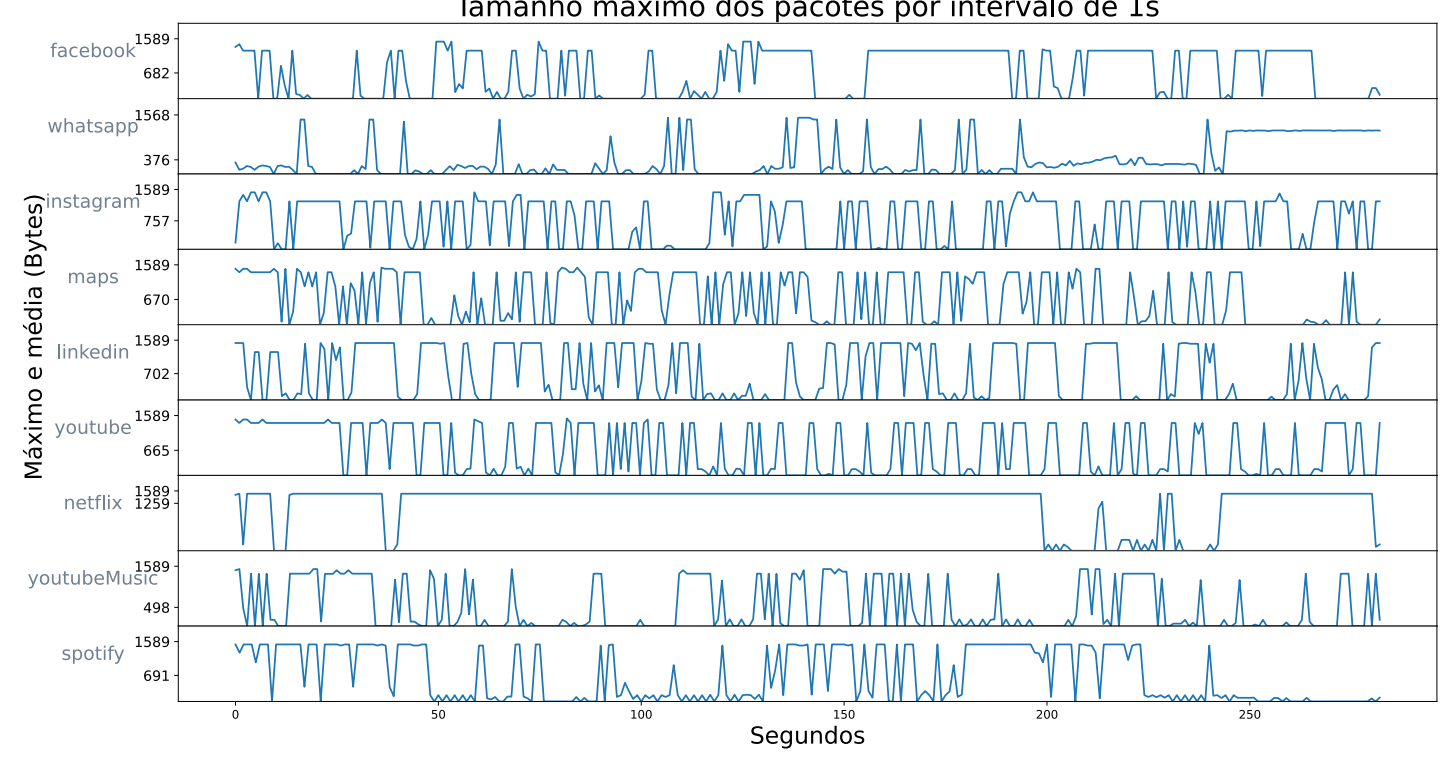

Figura 2. Tamanho máximo dos pacotes a cada intervalo de 1 segundo. São indicados o máximo e média de bytes durante todo o intervalo para cada aplicação. Todos os aplicativos possuem valor máximo de 1589 bytes por conta da existência de pacotes do TCP e de outros protocolos em todas as capturas, mesmo que pequena quantidade.

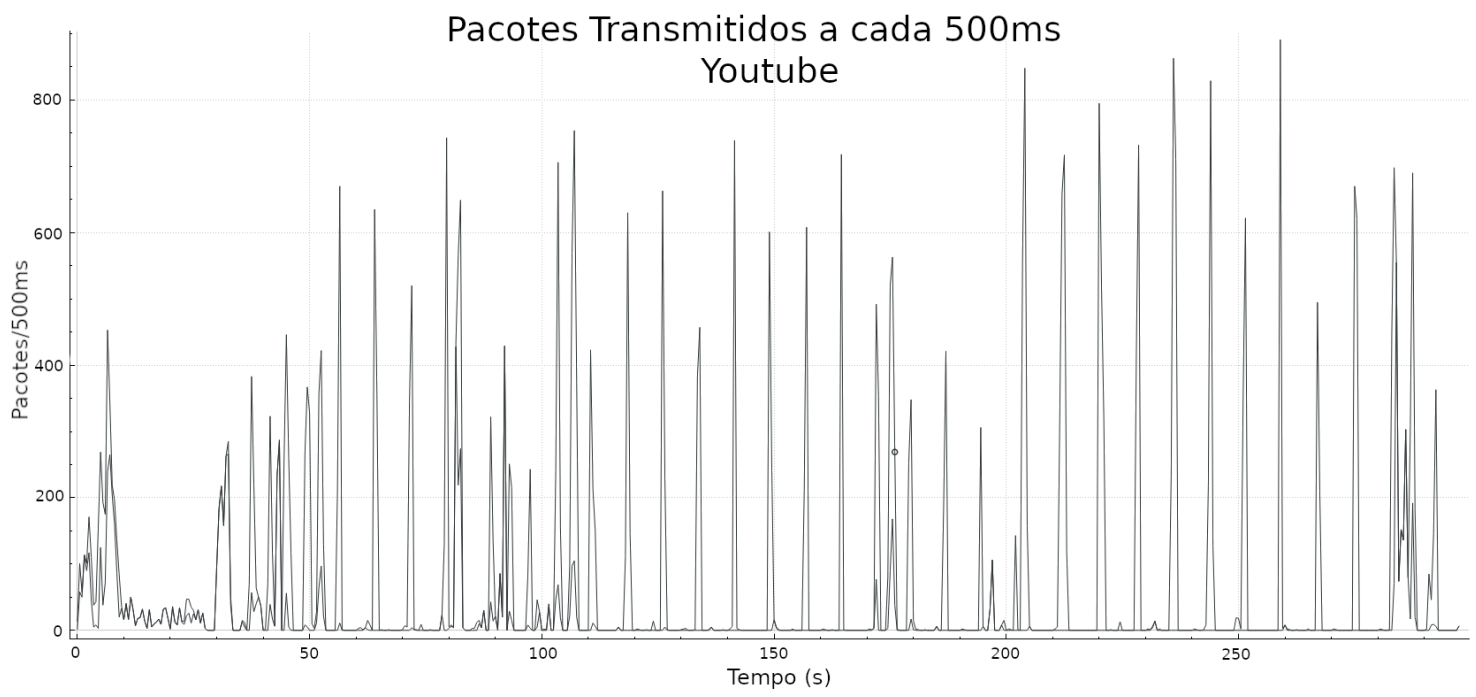

Figura 3. Número de pacotes transmitidos pelo Youtube por intervalo de $500 \mathrm{~ms}$. É possível observar as características de transmissão em rajadas.

com maior volume de tráfego de cada aplicação, na mesma configuração de rede na qual as capturas foram realizadas. As latências medidas, exibidas na Tabela 2, mostram que os aplicativos mantêm uma latência geralmente menor do que $20 \mathrm{~ms}$, sendo os aplicativos LinkedIn e Spotify desvios bastante grandes desta tendência. Isso pode ser explicado pela ausência de redes locais para distribuição de conteúdo dos serviços entregues por esses 

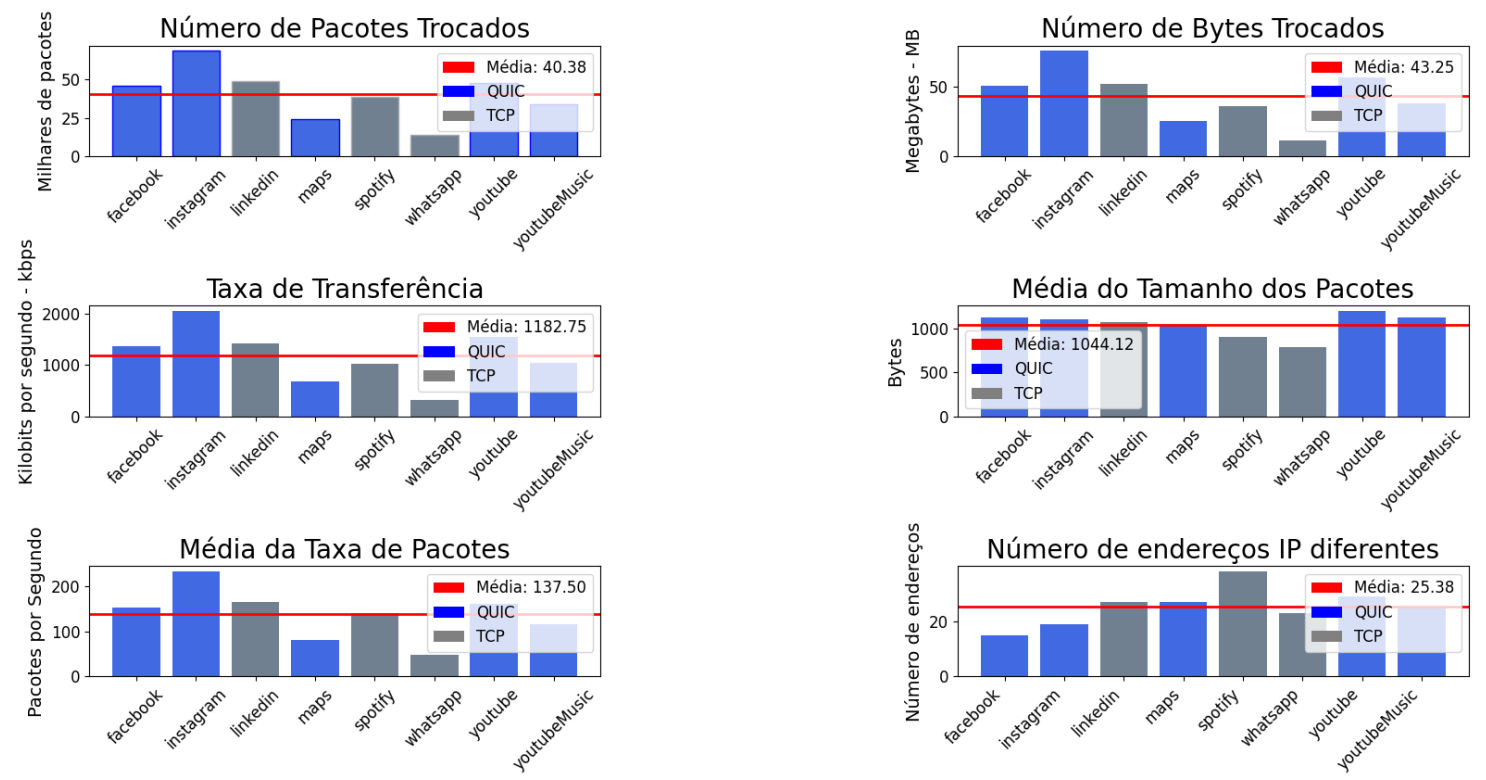

Figura 4. Estatísticas gerais do tráfego, incluindo a média das aplicações utilizando QUIC e TCP.

aplicativos.

\begin{tabular}{|c|c|c|}
\hline Aplicativo & Média (ms) & Desvio Padrão (ms) \\
\hline Facebook & 16,9 & 4,7 \\
\hline Instagram & 15,6 & 1,9 \\
\hline LinkedIn & 205,2 & 34,7 \\
\hline Google Maps & 14,6 & 2,5 \\
\hline Spotify & 319,7 & 34,0 \\
\hline WhatsApp Messenger & 14,1 & 1,9 \\
\hline Youtube & 14,8 & 2,2 \\
\hline Youtube Music & 14,3 & 2,2 \\
\hline \hline Mediana & $\mathbf{1 5 , 2}$ & \\
\hline
\end{tabular}

Tabela 2. Média e desvio padrão da latência dos endereços IPs de maior tráfego para cada aplicação.

Apesar de existirem variações significativas entre as estatísticas de redes para cada aplicação, foi possível obter diversos parâmetros aproximados para auxílio em simulações de redes, indicados na Tabela 3. O valor da latência foi extraído da mediana das latências dos oito aplicativos analisados, uma vez que esta medida permite expressar melhor a tendência do que a média, dado os valores discrepantes dos aplicativos LinkedIn e Spotify. O restante dos parâmetros foi extraído das médias para as estatísticas dos aplicativos, tal como indicado na Figura 4.

\section{Conclusões e Trabalhos Futuros}

A experimentação em redes de computadores por meio de simulação é bastante utilizada por conta da flexibilidade e do baixo custo desta técnica. Para se obter resultados significativos, é importante que os experimentos de simulação sejam os mais realistas possíveis. 


\begin{tabular}{|c|c|}
\hline Parâmetro & Quantidade/Característica \\
\hline Latência & $15,2 \mathrm{~ms}$ \\
\hline Número de Bytes & $43,25 \mathrm{MB}$ \\
\hline Taxa de Transferência & $1.182 \mathrm{Mbps}$ \\
\hline Taxa de Pacotes & 137,5 pacotes/s \\
\hline Tamanho dos pacotes & 1044,12 bytes \\
\hline Número de Endereços IP & 25,38 \\
\hline
\end{tabular}

\section{Tabela 3. Parâmetros obtidos através da média e mediana das estatísticas anali- sadas por comunicações de cinco minutos.}

Uma forma de obter esse realismo é por meio de medições prévias dos protocolos e aplicações que se deseja simular. Este artigo realiza essas medições focando em aplicações muito usadas em smartphones e buscando características relacionadas aos protocolos da camada de transporte, a fim de avaliar a aderência que as aplicações têm ao protocolo QUIC. Os resultados obtidos mostram que serviços de consumo de vídeos e imagens são os mais comuns novos adeptos do QUIC, com a ressalva de que o Netflix é uma exceção importante a esta tendência. Espera-se que as características dos tráfegos apresentadas neste artigo sejam úteis para pesquisas futuras em redes de computadores.

Como trabalho futuro, propõe-se a simulação das aplicações estudadas neste artigo em um ambiente de simulação de larga escala, com suporte à simulação de Internet móvel, a fim de avaliar como esses aplicativos afetarão as redes móveis de próxima geração (5G e $6 \mathrm{G})$.

\section{Agradecimentos}

Esta pesquisa é parte do INCT da Internet do Futuro para Cidades Inteligentes, financiado por CNPq (proc. 465446/2014-0), Coordenação de Aperfeiçoamento de Pessoal de Nível Superior - Brasil (CAPES) - Código de Financiamento 001 e FAPESP (procs. 14/509371 e 15/24485-9). Também é parte dos projetos FAPESP procs. 18/22979-2 e 20/02638-6.

\section{Referências}

Bhat, D., Rizk, A., and Zink, M. (2017). Not so QUIC: A Performance Study of DASH over QUIC. In Anais do NOSSDAV'17: 27th Workshop on Network and Operating Systems Support for Digital Audio and Video, páginas 13-18.

Bulgarella, F., Cociglio, M., Fioccola, G., Marchetto, G., and Sisto, R. (2019). Performance Measurements of QUIC Communications. In Anais do ANRW '19: Applied Networking Research Workshop, páginas 8-14.

Choi, J., Jung, C., Yeom, I., and Kim, Y. (2017). Streaming Service Enhancement on QUIC Protocol. In Anais do ICOMP'17: International Conference on Internet Computing, páginas 24-27.

Chromium (2013). Experimenting with QUIC. https://blog.chromium.org/ 2013/06/experimenting-with-quic.html. Último acesso em 28 de Junho de 2021. 
Couto, Y., Camarinha, D., and Batista, D. M. (2018). nsQUIC: Uma Extensão para Simulação do Protocolo QUIC no NS-3. In Anais do Salão de Ferramentas do SBRC 2018 .

Daigavhane, M. U. and Chawhan, M. (2018). Congestion Control Algorithm for TCP in Wireless Network. In Anais da RAIT'18: 4th International Conference on Recent Advances in Information Technology, páginas 1-4.

Iyengar, J. and Thomson, M. (2021). QUIC: A UDP-Based Multiplexed and Secure Transport. RFC 9000, RFC Editor.

Palmer, M., Krüger, T., Chandrasekaran, B., and Feldmann, A. (2018). The QUIC Fix for Optimal Video Streaming. In Anais do EPIQ'18: Workshop on the Evolution, Performance, and Interoperability of QUIC, páginas 43-49.

Postel, J. (1981). Transmission Control Protocol. STD 7, RFC Editor.

Qian, F., Wang, Z., Gerber, A., Mao, Z. M., Sen, S., and Spatscheck, O. (2011). Profiling Resource Usage for Mobile Applications: a Cross-Layer Approach. In Anais do MobiSys '11: 9th International Conference on Mobile systems, Applications, and Services, páginas 321-334.

Rüth, J., Poese, I., Dietzel, C., and Hohlfeld, O. (2018). A First Look at QUIC in the Wild. In Anais do PAM'18: Passive and Active Measurement.

Szabó, G., Rácz, S., Bezzera, D., Nogueira, I., and Sadok, D. (2016). Media QoE enhancement With QUIC. In Anais dos 2016 IEEE Conference on Computer Communications Workshops, páginas 219-220. 